Artículo científico

Volumen 30(2):313-331. Mayo-agosto, 2019 e-ISSN 2215-3608, doi:10.15517/am.v30i2.33938 https://revistas.ucr.ac.cr/index.php/agromeso/index

\title{
Percepciones de cambio climático y respuestas adaptativas de pequeños agricultores en dos paisajes guatemaltecos ${ }^{1}$
}

\section{Climate change perceptions and adaptive responses of small-scale farmers in two Guatemalan landscapes}

\author{
Bárbara Viguera², Francisco Alpízar², Celia A. Harvey³, M. Ruth Martínez-Rodríguez ${ }^{3,4}$, \\ Milagro Saborio-Rodríguez, Lucía Contreras $^{6}$
}

1 Recepción: 11 de julio, 2018. Aceptación: 25 de octubre, 2018. Este trabajo formó parte del proyecto Adaptación basada en Ecosistemas para pequeños productores de subsistencia y café en América Central (CASCADA) de la Iniciativa Internacional por el Clima (ICI), financiada por el Ministerio Federal de Medio Ambiente, Protección de la Naturaleza, Obras públicas y Seguridad Nuclear (BMUB) del Gobierno de la República de Alemania.

2 Centro Agronómico Tropical de Investigación y Enseñanza (CATIE). Apdo. 7170, Turrialba, Costa Rica. bviguera@ catie.ac.cr (http://orcid. org/0000-0002-5950-5113); falpizar@catie.ac.cr

3 Conservation International, Moore Center for Science. 2011 Crystal Drive, Suite 500, Arlington, VA, USA 22202. celiaharvey@stanfordalumni.org

4 Programa de las Naciones Unidas para el Ambiente (PNUMA), Oficina Regional para América Latina y el Caribe, Unidad de Cambio Climático.Panamá. ruth.martinez@un.org

5 Universidad de Costa Rica, Escuela de Economía e Instituto de Investigaciones en Ciencias Económicas, . Apdo 11501, San Pedro de Montes de Oca, Costa Rica. milagro.saborio@ucr.ac.cr

6 University of Sussex, Management and Economics, School of Businesses, Department of Economics. Reino Unido. mc662@sussex.ac.uk

\section{Resumen}

Introducción. Se espera que, a consecuencia del cambio climático, cultivos como el café (Coffea arabica L.), maíz (Zea mays) y frijol (Phaseolus vulgaris L.) sean menos productivos en América Central. Esto perjudicará la economía regional y los medios de vida de los pequeños productores, quienes dependen de estos cultivos para su seguridad alimentaria y bienestar. Es necesario entender cómo el cambio climático está afectando a los pequeños productores agrícolas en Guatemala para promover medidas que permitan enfrentar estos cambios. Objetivo. El objetivo de este trabajo fue caracterizar dos sistemas agrícolas de pequeña escala guatemaltecos, documentar cómo los pequeños productores perciben los impactos del cambio climático e identificar los esfuerzos de adaptación realizados. Materiales y métodos. Se realizaron encuestas estructuradas en hogares productores de café o granos básicos en los paisajes de Acatenango y Chiquimula, vulnerables al cambio climático, entre junio y julio de 2014. La investigación recogió las percepciones de los productores sobre cambios en temperatura y lluvia, los impactos reportados como consecuencia de dichos cambios y las acciones implementadas en las fincas a modo de respuesta. Resultados. Los resultados indicaron que el $95 \%$ de los encuestados percibió cambios en el clima en la última década y el $81 \%$ consideró que estos cambios afectaron negativamente su producción. El $41 \%$ de los productores implementaron prácticas para adaptarse a los cambios percibidos, en mayor medida aquellos que producen café. Las prácticas de adaptación implementadas en las fincas difirieron entre paisajes, cultivos y cambio percibido, siendo la más utilizada la siembra de árboles para amortiguar los efectos del incremento de temperatura. Conclusión. Para lograr una mayor respuesta adaptativa de los pequeños productores y para promover el uso de prácticas que incrementen su resiliencia, es necesario incrementar el apoyo técnico, financiero y político orientado a facilitar la adaptación de los pequeños productores ante el cambio climático.

Palabras clave: adaptación, café, granos básicos, manejo de finca, percepción de cambio climático. 


\begin{abstract}
Introduction. The productivity of certain crops such as coffee (Coffea arabica L.), maize (Zea mays) and beans (Phaseolus vulgaris L.) is expected to decline in Central America because of climate change. This will impact regional economies and livelihoods of smallholder farmers relying on these crops for their food security and livelihoods. There is a need to understand how climate change is already impacting smallholder farmers in Guatemala in order to promote adaptation measures that will allow them to face these impacts. Objective. The objectives of this study were to characterize two Guatemalan small-scale agricultural systems, describe farmers' perceptions of climate change and impacts, and document their adaptation efforts. Materials and methods. Structured surveys were carried out in households growing coffee or basic grains in Acatenango and Chiquimula landscapes, two climate change vulnerable landscapes, between June and July 2014. The research was based on farmers' perceptions of changes in temperature and rainfall, the impacts related to these changes, and the adaptation actions implemented in response to perceived changes. Results. Results indicated that $95 \%$ of farmers perceived changes in climate, and $81 \%$ of them considered these changes to have negatively affected their production. Only $41 \%$ of farmers had implemented measures to adapt to these changes, mainly those farmers growing coffee. The implemented adaptation measures differed between landscapes , crops, and usefulness against perceived change with tree planting being the most common adaptation practice to buffer against temperature increases. Conclusion. In order to improve the adaptive response ofsmallholder farmers and to promote the use of practices that increase resilience, it is neecessaryto provide more technical, financial and political support to facilitate the adaptation of small farmers facing climate change.
\end{abstract}

Keywords: basic grains, coffee, adaptation, farm management, climate change perception.

\title{
Introducción
}

Los modelos climáticos indican que la región centroamericana experimentará incrementos de temperatura y cambios en los patrones de precipitación en los próximos años (Imbach et al., 2017). Se espera además un aumento en la frecuencia y severidad de los eventos climáticos extremos (IPCC, 2012). La agricultura se verá fuertemente impactada por estos cambios (Hannah et al., 2017), en particular la agricultura de pequeña escala, por su dependencia de los recursos naturales y las limitantes socioeconómicas existentes para el mantenimiento de la producción (Morton, 2007). Por tanto, los cambios del clima comprometerán los medios de vida de miles de familias que dependen directa o indirectamente de la agricultura, afectando las economías de los países de la región centroamericana (CEPAL y CAC/SICA, 2014).

En Guatemala se observaron cambios en las tendencias de temperatura y precipitación en el periodo comprendido entre 2001 y 2014), respecto al periodo de 1970 a 2000, con diferencias entre regiones. En particular, se reportaron incrementos de temperatura entre 0,2 y $10,3 \%$, e incrementos de precipitación entre 2,3 y $48 \%$ con distribución irregular a lo largo del año; si bien esta tendencia podría estar influenciada por el incremento de eventos ciclónicos en buena parte del país (MARN, 2015), se espera que, a consecuencia de estos cambios, y los pronósticos de cambio en las variables climáticas y la disponibilidad de agua bajo diferentes escenarios (Imbach et al., 2012; 2015), el rendimiento de los cultivos de maíz, frijol y otros granos básicos se reduzca significativamente en las próximas décadas (Hannah et al., 2017). Esto afectará los medios de vida y la seguridad alimentaria de la población vinculada a la producción de granos básicos (Eitzinger et al., 2013), así como las oportunidades laborales en el ámbito rural y el crecimiento económico, influenciados por ciertos cultivos como el café (CEPAL y CAC/SICA, 2014).

Aunque se han identificado cambios en los patrones climáticos, se desconoce cómo se traducen estos cambios en la agricultura, cómo afectan a los pequeños productores y qué estrategias de adaptación son las más apropiadas para minimizar los impactos (Donatti et al., 2018). Conocer las percepciones de los pequeños productores sobre los 
cambios e impactos del clima en sus cultivos, así como las estrategias que están implementando en respuesta a estos, es un insumo relevante para promover la adaptación del sector agrícola a los cambios en temperatura y patrones de precipitación, a través de medidas eficientes de adaptación (Galindo et al., 2014).

Entre las diversas opciones de adaptación existentes, Noble et al. (2014) mostraron una lista detallada de medidas de adaptación, en la cual se prestó especial atención a las prácticas de adaptación basada en ecosistemas (AbE) implementadas a nivel de finca. La adaptación basada en ecosistemas consiste en el uso de la biodiversidad y los servicios ecosistémicos para incrementar la resiliencia de los sistemas frente al cambio climático (CBD, 2009). Las medidas de $\mathrm{AbE}$ son costo-efectivas, proporcionan beneficios climáticos y co-beneficios de tipo ambiental, social y económico, que mejoran los medios de vida de los pequeños productores (Vignola et al., 2015).

Entender el proceso de adaptación de los pequeños productores contribuye a orientar las políticas públicas, los programas de extensión y las iniciativas de desarrollo hacia las necesidades de estos, para lograr unos sistemas agrícolas adaptados a los cambios del clima, más resilientes social, ambiental, y económicamente (Donatti et al., 2017).

El objetivo de este trabajo fue caracterizar dos sistemas agrícolas de pequeña escala guatemaltecos, documentar cómo los pequeños productores perciben los impactos del cambio climático e identificar los esfuerzos de adaptación realizados.

\section{Materiales y métodos}

\section{Paisajes de estudio}

Se seleccionaron dos sistemas productivos representativos de la agricultura de pequeña escala de Guatemala: el café (Coffea arabica) y los granos básicos (principalmente maíz [Zea mays] y frijol [Phaseolus sp.]), en dos paisajes guatemaltecos vulnerables al cambio climático: Acatenango, en el altiplano, y Chiquimula, en el Corredor Seco Centroamericano. La selección de los paisajes de estudio se realizó con base en los siguientes criterios: (i) predominio de agricultura de pequeña escala, (ii) presencia de café y/o granos básicos como cultivo principal, (iii) elevada vulnerabilidad al cambio climático y (iv) baja capacidad adaptativa. La metodología para identificar los paisajes vulnerables al cambio climático se describió en Holland et al. (2017).

El paisaje Acatenango está situado en el altiplano, al suroeste del país; está conformado por los municipios de Acatenango, San Pedro Yepocapa (pertenecientes al departamento de Chimaltenango) y Alotenango (departamento de Sacatepéquez), que juntos suman $484 \mathrm{~km}^{2}$ de extensión. El clima es templado, con temperaturas medias en torno a los $20{ }^{\circ} \mathrm{C}$ y precipitación anual entre 1700 y $2400 \mathrm{~mm}$ (Climate-Data.org, 2018), que da lugar a zonas climáticas de bosque muy húmedo subtropical y bosque húmedo montano bajo subtropical (Holdridge, 1947). Los suelos son arenosos, fértiles, con pendiente alta y muy susceptibles a la erosión hídrica y eólica. El paisaje es atravesado por el macizo volcánico Fuego-Acatenango.

El paisaje Chiquimula está situado al sureste de Guatemala, en la demarcación política trinacional Trifinio, y forma parte del Corredor Seco Centroamericano. Está conformado por los municipios de San Juan Ermita, San Jacinto y Quezaltepeque (departamento de Chiquimula), que en conjunto suman $386 \mathrm{~km}^{2}$ de extensión. Presenta climas de bosque húmedo subtropical y de bosque seco subtropical (Holdridge, 1947), con estación lluviosa de mayo a octubre, con temperaturas medias en torno a $24,5^{\circ} \mathrm{C}$ y precitación de $1100 \mathrm{~mm}$ (Climate-Data.org, 2018). Chiquimula es predominantemente agrícola. El relieve de Chiquimula es montañoso irregular con alturas que van desde $\operatorname{los} 435$ a los $1350 \mathrm{msnm}$. 


\section{Muestreo y recolección de datos}

La información que se presenta en este estudio fue recolectada en junio y julio de 2014. Ante la falta de información sobre la distribución de la población en el paisaje para diseñar el muestreo de productores, se generó información secundaria a partir del mapeo de los techos por medio de identificación visual con ortofotografía aérea. Se identificaron 15712 y 13745 techos en Acatenango y Chiquimula, respectivamente. Se consideró que cada techo correspondía a una unidad familiar. Se seleccionaron aleatoriamente 150 hogares agrícolas en cada paisaje, según criterios de un estudio realizado a nivel regional. Posteriormente, con ayuda de un dispositivo de georreferenciación (GPS), se visitaron los hogares seleccionados para aplicar la encuesta y se sustituyeron en caso de que el techo no correspondiese a un hogar agrícola (hogar no agrícola, casa abandonada u otro tipo de edificación). En cada hogar se encuestó al cabeza de familia, es decir, la persona con capacidad de tomar decisiones sobre el manejo de la finca.

Las unidades de estudio fueron hogares de pequeños productores agrícolas de ambos paisajes. Debido a la falta de acuerdo sobre la definición de pequeño productor (Conway, 2011), se encuestaron todos los hogares agrícolas aleatoriamente seleccionados y posteriormente, se eliminaron las observaciones de productores cuyo tamaño de finca era superior a la media del tamaño de finca más dos veces la desviación estándar (calculado para cada paisaje y cultivo). Los resultados que se presentan corresponden a los pequeños productores encuestados y sus hogares, un total de 149 jefes de hogar en Acatenango (114 hombres, 35 mujeres) y 115 en Chiquimula ( 85 hombres, 30 mujeres).

La encuesta fue validada por medio de grupos focales con miembros de las comunidades donde se llevó a cabo la misma, pilotada en campo y aprobada por el Comité de Revisión Ética Interno de Conservación Internacional. De esta forma se verificó que la encuesta siguiera la normativa institucional para la participación libre e informada de los productores (Buppert y McKeehan, 2013). La encuesta se implementó en persona por un equipo de encuestadores entrenados y un supervisor de campo. Se utilizaron dispositivos electrónicos (tabletas) y el software Survey-CTO, para recolectar los datos e incrementar el nivel de confianza de los mismos por medio de controles de seguridad automáticos que monitoreaban el avance y la coherencia de las respuestas.

\section{Información recolectada y análisis realizados}

La información que se describe en este estudio fue recolectada por medio de una encuesta estructurada, que contenía 553 preguntas de selección única o selección múltiple (Alpizar et al., 2019). Para describir los sistemas agrícolas de los paisajes, se realizaron preguntas sobre las características socioeconómicas de los jefes de hogar, de los hogares y de aspectos del sistema productivo. En este documento se describen los resultados obtenidos con Stata 13.

Para caracterizar el manejo de las fincas, se hicieron preguntas sobre el uso de un conjunto amplio de prácticas de manejo: chapias, uso de agroquímicos, poda, deshija y otros en el caso del café, densidad de siembra, uso del fuego, riego y uso de agroquímicos en el caso del maíz y frijol. Se preguntó además sobre las variedades de café utilizadas y el acceso a mercado de los granos básicos. Estas preguntas se hicieron a cada entrevistado que cultivaba café, granos básicos o ambos cultivos. Las preguntas de otras secciones se formularon solamente a aquellos encuestados que dieron una respuesta específica a una pregunta previa o pregunta de seguimiento (Cuadro 1).

Se preguntó a los encuestados sobre el uso de ciertas prácticas de manejo (consideradas de adaptación basadas en ecosistemas), por su utilidad para lidiar con el cambio climático. Con los productores de café se recolectó información de las siguientes prácticas de Adaptación basada en Ecosistemas (AbE): surcos a contorno, cercas vivas, uso de sombra y chapias manuales. A los productores de granos básicos, se les preguntó sobre el uso de surcos a contorno, cercas vivas, árboles dispersos, no quema de rastrojos (evitar el uso de fuego como práctica de manejo), uso de rastrojo o restos de cosecha como cobertura muerta y labranza mínima para los cultivos de maíz y frijol. Para cada práctica, se realizaron preguntas de seguimiento sobre el uso, año de implementación y beneficios percibidos del uso de la práctica, a aquellos encuestados que reportaron su uso. La caracterización de los sistemas productivos 
Cuadro 1. Descripción de la información que se presenta en los cuadros y figuras del documento, donde se indica el subgrupo de encuestados que respondió cada grupo de pregunta y el número de observaciones (tamaño de la muestra en función del grupo que responde), la información que se presenta y la ubicación de la misma en el documento. Paisajes de Acatenango y Chiquimula, Guatemala. 2014.

Table 1. Description of information contained in tables and figures of this document, indicating the subgroup that answered each set of questions, the number of observations (sample size depending on the group of respondents), and the information that each table contains. Acatenango and Chiquimula landscapes, Guatemala. 2014.

\begin{tabular}{|c|c|c|c|c|}
\hline \multirow{2}{*}{ Quién responde la pregunta } & \multirow{2}{*}{ Qué información se presenta } & \multicolumn{2}{|c|}{ N observaciones } & \multirow{2}{*}{ Ubicación } \\
\hline & & Acatenango & Chiquimula & \\
\hline Todos los productores & Caracterización de jefes, hogares y fincas & 149 & 115 & Cuadro 2 \\
\hline Todos los productores & Manejo de la finca & 149 & 115 & Texto \\
\hline $\begin{array}{l}\text { Todos los productores de café; aquellos que } \\
\text { cambian de variedad }\end{array}$ & $\begin{array}{l}\text { Variedades de café } . . . \ldots \ldots \ldots \ldots . . . . . \\
\text { Cambio de variedades........... }\end{array}$ & $\begin{array}{c}112 \\
34 \\
\end{array}$ & $\begin{array}{c}17 \\
4\end{array}$ & Cuadro 3 \\
\hline $\begin{array}{l}\text { Productores de café que usan cada práctica } \\
\text { contestan las preguntas de seguimiento }\end{array}$ & $\begin{array}{l}\text { Beneficios del uso de prácticas AbE en café } \\
\text { - Surcos a contorno............. } \\
\text { - Cercas vivas................... } \\
\text { - Sombra....................... } \\
\text { - Chapias manuales........... }\end{array}$ & $\begin{array}{c}52 \\
61 \\
111 \\
111\end{array}$ & $\begin{array}{c}6 \\
9 \\
16 \\
17\end{array}$ & Fig. 1 \\
\hline Todos los productores de café & Daños observados en café & 112 & 17 & Fig. 2 \\
\hline $\begin{array}{l}\text { Los productores de granos básicos sobre el uso } \\
\text { de AbE }\end{array}$ & $\begin{array}{l}\text { Beneficios del uso de prácticas AbE en granos } \\
\text { básicos } \\
\text { - Surcos a contorno........... } \\
\text { - Cercas vivas................ } \\
\text { - Árboles dispersos........... } \\
\text { - No quema................... } \\
\text { - Uso de rastrojo............. } \\
\text { - Labranza mínima........... }\end{array}$ & $\begin{array}{l}38 \\
18 \\
32 \\
81 \\
72 \\
60\end{array}$ & $\begin{array}{l}45 \\
19 \\
66 \\
89 \\
45 \\
65\end{array}$ & Fig. 3 \\
\hline Productores de maíz y frijol & $\begin{array}{l}\text { Problemas de producción en: } \\
- \text { Maíz........................... } \\
\text { - Frijol......................... }\end{array}$ & $\begin{array}{l}89 \\
64\end{array}$ & $\begin{array}{l}114 \\
108\end{array}$ & Fig. 4 \\
\hline $\begin{array}{l}\text { Productores que perciben cambios en la } \\
\text { temperatura o la lluvia }\end{array}$ & $\begin{array}{l}\text { Cambios percibidos en: } \\
\text { - Temperatura } \ldots \ldots \ldots \ldots \ldots \ldots \ldots \\
\text { - Lluvia......................... }\end{array}$ & $\begin{array}{l}132 \\
127\end{array}$ & $\begin{array}{l}101 \\
110\end{array}$ & Cuadro 4 \\
\hline $\begin{array}{l}\text { Solo los productores que reportaron que cambios } \\
\text { en el clima han afectado su producción }\end{array}$ & 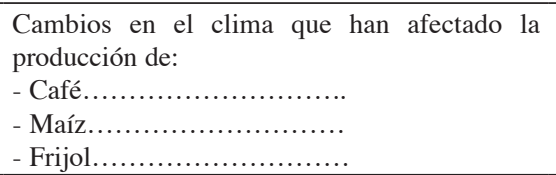 & $\begin{array}{l}73 \\
58 \\
44\end{array}$ & $\begin{array}{c}8 \\
99 \\
85\end{array}$ & Cuadro 5 \\
\hline $\begin{array}{l}\text { Solo los productores que reportaron haber } \\
\text { implementado cambios de manejo en respuesta } \\
\text { a los cambios percibidos en el clima }\end{array}$ & $\begin{array}{l}\text { Prácticas de manejo de finca implementadas en } \\
\text { respuesta a cambios percibidos en temperatura } \\
\text { y lluvia. }\end{array}$ & 73 & 27 & Cuadro 6 \\
\hline
\end{tabular}

de café y granos básicos concluyó con una pregunta sobre los problemas que se han experimentado en producción de café, maíz y frijol. Esta pregunta de respuesta múltiple fue formulada a todos los productores, algunos encuestados reportaron no tener ningún problema en sus cultivos, por lo que las respuestas se presentan para aquellos productores que sí reportaron problemas (Cuadro 1).

También se indagó sobre la percepción de los encuestados sobre cambios en el clima. Se preguntó específicamente a cada encuestado si percibió cambios en temperatura en los diez años anteriores a la encuesta. En caso afirmativo, se preguntaba: (a) cuáles fueron los cambios percibidos, y (b) si se había implementado alguna variación en el manejo de la finca debido a las percepciones de fluctuación en temperatura. Si la respuesta a la segunda pregunta era afirmativa, se preguntaba sobre cuáles cambios de manejo de finca fueron implementados (Cuadro 1). El proceso 
se repitió en caso de percepción de variaciones en los patrones de lluvia. Además, se preguntó a los productores sobre cuáles cambios del clima (incluyendo temperatura, lluvia o en la ocurrencia de eventos hidrometeorológicos extremos) relacionan con impactos negativos en producción de café, maíz y frijoles. El Cuadro 1 refleja la cantidad de observaciones en cada una de estas preguntas de seguimiento, por paisaje. La información recolectada corresponde a percepciones de productores encuestados y se describe por medio de tablas e indicadores estadísticos.

\section{Resultados}

\section{Caracterización de productores y fincas}

De los 149 productores encuestados en Acatenango, el 37,6 \% eran productores de café, el 37,6 \% producían granos básicos (maíz y/o frijol) y el 24,8 \% restante manejaban ambos cultivos. En Chiquimula, de los 115 productores de la muestra, el 85,2\% cultivaba granos básicos y el 14,8\% restante combinaba los granos básicos con la producción de café. En el Cuadro 2 se resumen las características de las fincas, del jefe de hogar, del hogar y los indicadores de diversificación y riqueza de los hogares en ambos paisajes, en función de su actividad productiva.

Cuadro 2. Características de las fincas, del jefe de hogar, del hogar y de los recursos de pequeños productores de café y granos básicos en Acatenango $(\mathrm{n}=149)$ y Chiquimula $(\mathrm{n}=115)$. Guatemala. 2014.

Table 2. Characteristics of the farms, household heads and resources of smallholder coffee and basic grain farmers in Acatenango $(\mathrm{n}=149)$ and Chiquimula ( $\mathrm{n}=115)$. Guatemala, 2014.

\begin{tabular}{|c|c|c|c|c|c|c|c|c|c|c|}
\hline \multirow[b]{3}{*}{ Observaciones } & \multicolumn{6}{|c|}{ Acatenango } & \multicolumn{4}{|c|}{ Chiquimula } \\
\hline & \multicolumn{2}{|c|}{ Café } & \multicolumn{2}{|c|}{ Granos básicos } & \multicolumn{2}{|c|}{$\begin{array}{l}\text { Café y granos } \\
\text { básicos }\end{array}$} & \multicolumn{2}{|c|}{ Granos básicos } & \multicolumn{2}{|c|}{$\begin{array}{c}\text { Café y granos } \\
\text { básicos }\end{array}$} \\
\hline & $X=56$ & DE & $X=37$ & DE & $X=56$ & DE & $X=98$ & DE & $X=17$ & DE \\
\hline \multicolumn{11}{|l|}{ Características de la finca } \\
\hline Tamaño finca (ha) & 1,1 & $\pm 1,1$ & 0,6 & $\pm 0,3$ & 1,6 & $\pm 1,4$ & 1,2 & $\pm 1,1$ & 1,6 & $\pm 1,1$ \\
\hline Número de parcelas en la finca (n) & 1,4 & $\pm 0,7$ & 1,5 & $\pm 0,6$ & 2,4 & $\pm 1,1$ & 1,4 & $\pm 0,7$ & 2,4 & $\pm 1,1$ \\
\hline Distancia de la finca al hogar $(\mathrm{km})$ & 4,7 & $\pm 4,6$ & 6,1 & $\pm 6,4$ & 5,3 & $\pm 4,6$ & 2,9 & $\pm 3,9$ & 1,7 & $\pm 1,4$ \\
\hline Altitud (msnm) & 1334 & \pm 256 & 1677 & \pm 366 & 1426 & \pm 265 & 816 & \pm 253 & 1093 & \pm 236 \\
\hline Presencia de bosque (\%) & 7,3 & & 0,0 & & 8,9 & & 12,2 & & 29,4 & \\
\hline Cantidad de bosque (ha) & 0,2 & $\pm 0,2$ & - & - & 0,7 & $\pm 0,5$ & 0,8 & $\pm 0,5$ & 1,4 & $\pm 2,4$ \\
\hline \multicolumn{11}{|l|}{ Características del jefe de hogar } \\
\hline Jefatura femenina $(\%)$ & 23,2 & & 24,3 & & 23,2 & & 26,5 & & 23,5 & \\
\hline Edad (años) & 49,7 & $\pm 16,5$ & 45,2 & $\pm 15,9$ & 48,6 & $\pm 13,3$ & 45,4 & $\pm 13,6$ & 49 & $\pm 14,2$ \\
\hline Experiencia en agricultura (años) & 28,3 & $\pm 18,2$ & 27,5 & $\pm 15,8$ & 31,5 & $\pm 13,8$ & 29,0 & $\pm 14,7$ & 33,5 & $\pm 14,2$ \\
\hline Sin educación formal (\%) & 21,4 & & 29,7 & & 28,6 & & 29,6 & & 29,4 & \\
\hline \multicolumn{11}{|l|}{ Características del hogar } \\
\hline Tamaño del hogar (miembros) & 5,1 & $\pm 2,1$ & 5,5 & $\pm 3,0$ & 5,6 & $\pm 2,4$ & 5.0 & $\pm 2,1$ & 5.3 & $\pm 2,5$ \\
\hline Hogares con migrantes permanentes (\%) & 8,9 & & 13,5 & & 10,7 & & 11,2 & & 23,5 & \\
\hline Trabajo en finca ( $\mathrm{n}$ personas) & 1,7 & $\pm 1,1$ & 2,1 & $\pm 1,3$ & 2 & $\pm 1,1$ & 1.6 & $\pm 0,9$ & 2.4 & $\pm 1,9$ \\
\hline Trabajo fuera de finca ( $\mathrm{n}$ personas) & 1,2 & $\pm 1,0$ & 1,9 & $\pm 1,7$ & 1,7 & $\pm 1,5$ & 0,8 & $\pm 1,0$ & 1.2 & $\pm 1,1$ \\
\hline Tiene ganadería $(\%)$ & 7,1 & & 32,4 & & 8,9 & & 14,3 & & 29,4 & \\
\hline Tiene aves de patio $(\%)$ & 39,3 & & 59,5 & & 58,9 & & 80,6 & & 76,5 & \\
\hline Tiene huerto casero $(\%)$ & 7,1 & & 18,9 & & 9,1 & & 13,3 & & 17,8 & \\
\hline Tiene carro o motocicleta (\%) & 32,1 & & 13,5 & & 26,8 & & 20,4 & & 29,4 & \\
\hline Pertenece a organización (\%) & 41,1 & & 13,5 & & 20,0 & & 21,4 & & 47,1 & \\
\hline
\end{tabular}

X: Media; DE: desviación estándar / X: Mean; DE: standard deviation. 


\section{Manejo de los cafetales, uso de prácticas de AbE y problemas de producción}

El café es un cultivo perenne que requiere manejo y aportes externos en varias fases del cultivo. En promedio, en los cafetales de Acatenango se realizaban tres chapias, 1,9 aplicaciones de fertilizante, 1,5 de fungicida, 0,9 de herbicida y se deshijaba 0,5 veces por año. El $15 \%$ de las fincas de café tenían algún tipo de certificación ambiental y el $5 \%$ producían café orgánico. La mayor parte de los productores (62\%) entregaban su café a un comprador independiente, otros trabajaban con cooperativas $(21 \%)$ y beneficios privados $(13 \%)$. Las variedades de café más cultivadas eran Caturra, Catuai, Bourbón y Catimor (Cuadro 3). Solo el $31 \%$ de los caficultores informaron haber cambiado de variedad en los últimos años, y realizaron este cambio para incrementar la resistencia de los cafetales a plagas y enfermedades.

Cuadro 3. Variedades de café cultivadas en las fincas de Acatenango y Chiquimula, y razones de cambio de variedades. 2004 - 2014. Guatemala.

Table 3. Coffee varieties cultivated in Acatenango and Chiquimula farms, and reasons for changing varieties. 2004 - 2014. Guatemala.

\begin{tabular}{|c|c|c|}
\hline & Acatenango & Chiquimula \\
\hline & $\mathrm{N}=112$ & $\mathrm{~N}=17$ \\
\hline Variedades promedio por finca & 1,99 & 1,64 \\
\hline Tiene $>1$ variedad & $59 \%$ & $46 \%$ \\
\hline Tiene $>2$ variedades & $23 \%$ & $14 \%$ \\
\hline \multicolumn{3}{|l|}{ Variedades de café sembradas } \\
\hline Caturra & $48 \%$ & $50 \%$ \\
\hline Catuai & $38 \%$ & $25 \%$ \\
\hline Catimor & $25 \%$ & $29 \%$ \\
\hline Typica & $7 \%$ & $36 \%$ \\
\hline Bourbon & $32 \%$ & $0 \%$ \\
\hline Costa Rica 95 & $18 \%$ & $4 \%$ \\
\hline Pache & $10 \%$ & $7 \%$ \\
\hline Otros (Icatú, Castillo, Sarchimor, etc.) & $18 \%$ & $11 \%$ \\
\hline Razones de cambio de variedad & $\mathrm{N}=34$ & $\mathrm{~N}=4$ \\
\hline Resistencia a plagas o enfermedades & $82 \%$ & $50 \%$ \\
\hline Aumentar productividad & $26 \%$ & $75 \%$ \\
\hline Resistencia a frío & $3 \%$ & $25 \%$ \\
\hline Razones de mercado & $3 \%$ & $0 \%$ \\
\hline Resistencia a sequía & $3 \%$ & $0 \%$ \\
\hline Recibió incentivo para cambiar & $0 \%$ & $0 \%$ \\
\hline
\end{tabular}

$\mathrm{N}=$ tamaño de la muestra / $\mathrm{N}=$ sample size.

En Chiquimula, donde el cultivo de café ha sido recientemente introducido (en contraste con Acatenango, donde existe tradición cafetalera), el manejo es menos intensivo; en promedio se realizaban 2,4 chapias al año, 1,4 aplicaciones de fertilizante, 0,7 de fungicida, 0,2 de herbicida y solamente un productor realizaba deshija. El $35 \%$ sembraba el café en asocio con otros cultivos. La mayor parte de los productores (88\%) entregaban su café a un comprador independiente y el resto en beneficios privados (12\%). La mitad de los productores solamente manejaba una variedad en el cafetal. Caturra era la variedad de café más usada en la zona (35\%), seguida por Typica, 
Catimor, Catuai (Cuadro 3). Tan solo cuatro productores realizaron cambios de variedad en la última década. Ningún productor recibió incentivos para realizar cambio de variedades de café.

En los dos paisajes de estudio se manejaba el café con sombra, con variaciones en composición y estructura entre paisajes. En Acatenango era más frecuente ver cafetales con un único estrato de sombra, mientras que en Chiquimula la sombra tendió a ser más compleja, en forma de policultivos comerciales, policultivos tradicionales y sombra rústica (café sembrado bajo dosel arbóreo natural, generalmente compuesto por muchas especies), que presentaban mayor diversidad de especies y varios niveles de dosel. En Acatenango el $41 \%$ de las fincas manejaban una especie de sombra, el $33 \%$ dos y el $25 \%$ tres o más especies. Entre las especies más comunes se encontraban la grevillea (Grevillea robusta), el chalum (Inga xalapensis), otras ingas y fabáceas. Las especies acompañantes eran mayoritariamente frutales, entre ellas el aguacate (Persea americana) y cítricos (Citrus sp.), así como otras especies propias de la zona. En Chiquimula, el $79 \%$ de los productores manejaba sombra diversificada. La mayoría tenían combinaciones de frutales: mango (Mangifera indica), musáceas (Musa sp.), anona (Anona sp.), manzana (Malus sp.), y cacao (Theobroma cacao), entre otras. Muy pocos productores tenían especies maderables como pino (Pinus sp.), roble (Quercus copeyensis), cedro (Cedrela odorata) o con otros usos como el guachipelín (Diphysa americana).

El uso de árboles de sombra, el manejo de la sombra y las chapias eran las prácticas de Adaptación basada en Ecosistemas más frecuentes en los cafetales de ambos paisajes. La mayoría de estas prácticas eran utilizadas tradicionalmente. Los productores percibían beneficios del uso de las prácticas, principalmente de tipo adaptativas y mejoramiento de las condiciones del suelo (Figura 1). Los beneficios percibidos eran similares entre paisajes.

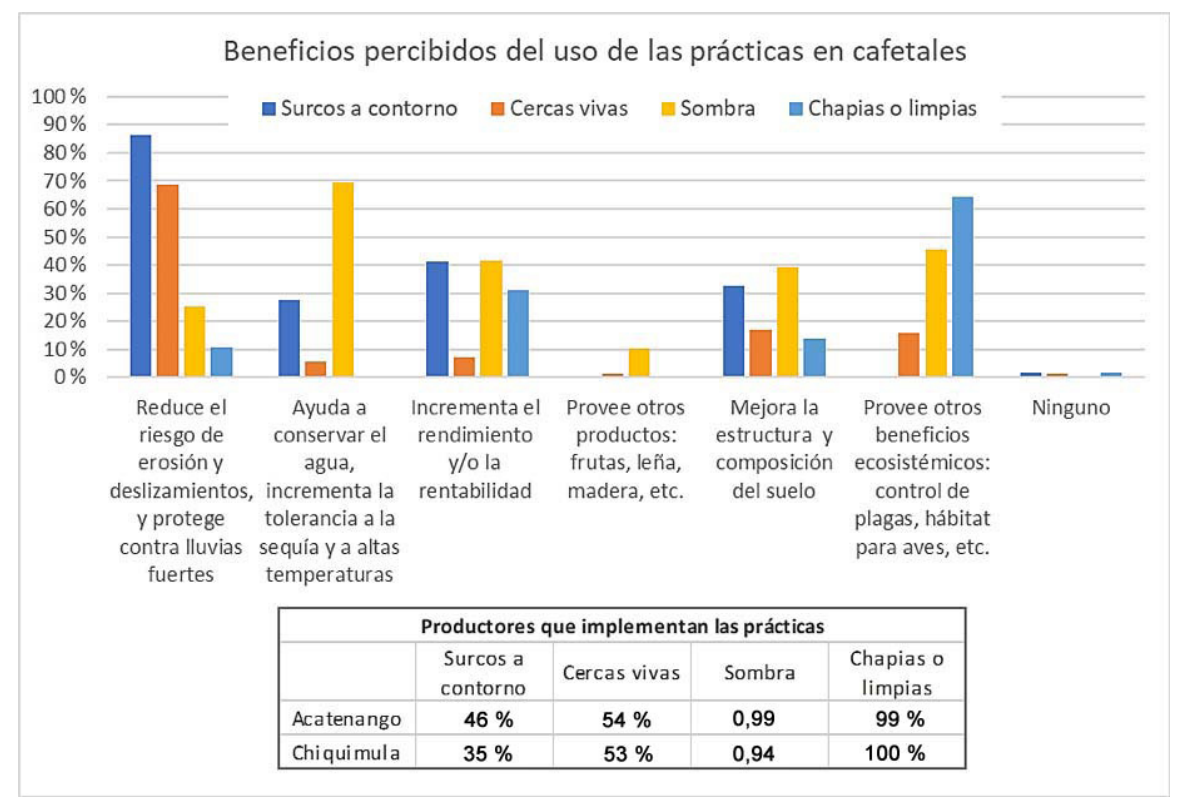

Figura 1. Beneficios del uso de las prácticas de Adaptación basada en Ecosistemas (AbE) percibidos por los productores de café en Acatenango y Chiquimula. Guatemala, 2014.

Figure 1. Benefits of using Ecosystem-based Adaptation (EbA) practices perceived by coffee farmers in Acatenango and Chiquimula. Guatemala, 2014.

El $96 \%$ de los cafetaleros de Acatenango y todos los cafetaleros encuestados en Chiquimula habían observado problemas de diverso tipo sobre sus plantaciones. En ambos paisajes el daño observado con más frecuencia eran las 
enfermedades, particularmente la roya del cafeto (Hemileia vastatrix) y en menor medida el ojo de gallo (Mycena citricolor). Otros daños importantes eran la caída de las flores y, en Chiquimula, la pérdida de granos y la muerte de plantas (Figura 2).

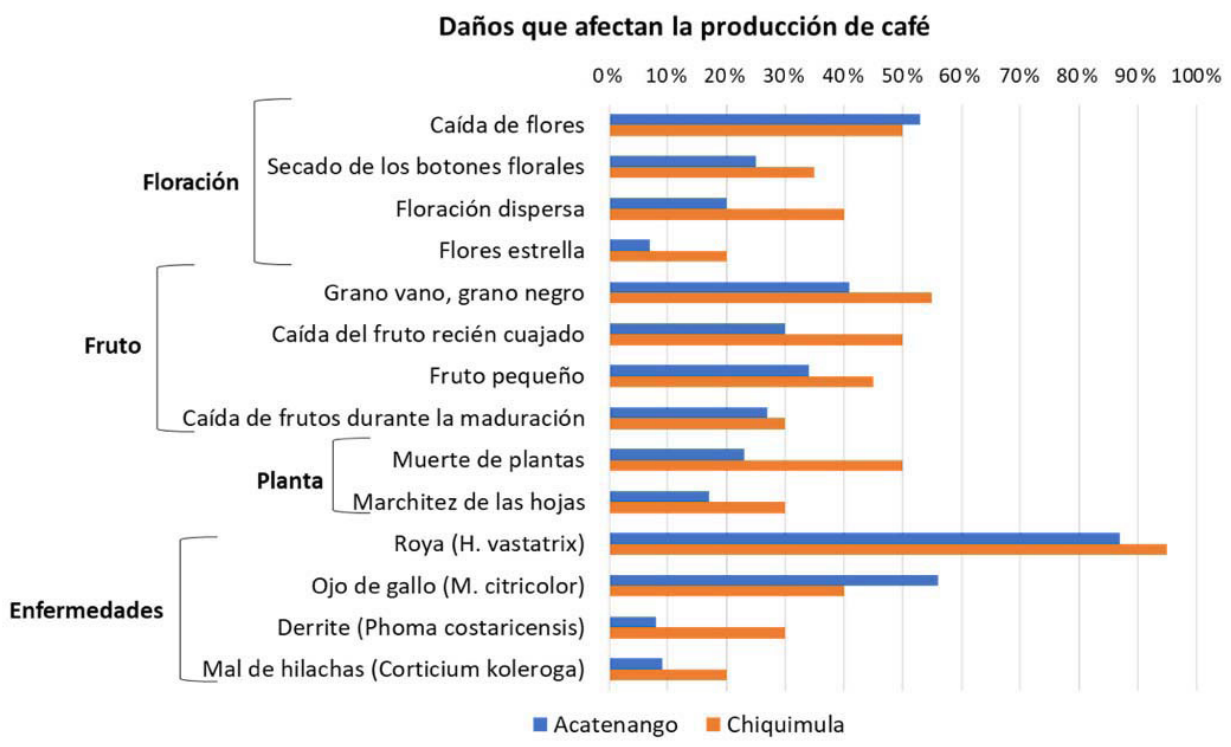

Figura 2. Porcentaje de productores que observaron distintos problemas o daños en sus cafetales, en Acatenango ( $\mathrm{n}=112$ ) y Chiquimula $(\mathrm{n}=17)$, Guatemala. 2004-2014.

Figure 2. Percent of coffee farmers who have observed different types of problems or damage in their coffee farms in Acatenango $(\mathrm{n}=112)$ and Chiquimula (n=17), Guatemala. 2004-2014.

\section{Manejo de los granos básicos, uso de prácticas de adaptación basada en ecosistemas (AbE) y problemas de producción}

Los cultivos de granos básicos se manejan diferente entre los paisajes estudiados. En promedio en Acatenango, las milpas se manejaban en densidades de 10000 plantas.ha $^{-1}$, el frijol a 33000 plantas.ha $^{-1}$, se obtenían 1,7 cosechas de maíz y 1 de frijol al año, con productividades de 1,8 t.ha $^{-1}$ para el maíz y 0,8 t.ha-1 para el frijol. Un $13 \%$ de los productores utilizaba el fuego para preparar la siembra y el $3 \%$ regaban sus plantíos. En cuanto a insumos, la mayoría (89\%) aplicaba fertilizantes sintéticos y el $31 \%$ fertilizantes orgánicos. El $28 \%$ usaba pesticidas y el $44 \%$ fungicidas.

En Chiquimula se obtenían en promedio una cosecha de maíz y una de frijol al año. Las milpas se manejaban a mayor densidad que en Acatenango, 22000 plantas.ha $^{-1}$ el maíz y 38000 plantas.ha $^{-1}$ el frijol, y obtenían menor productividad: 0,9 t.ha ${ }^{-1}$ de maíz y 0,6 t.ha ${ }^{-1}$ de frijol. El $19 \%$ de los productores usaba fuego para preparar la siembra y el $3 \%$ regaban sus plantíos. En cuanto a insumos, la mayoría (89\%) aplicaba fertilizantes sintéticos y un porcentaje menor (14 \%) aplica fertilizantes orgánicos. En Chiquimula aplicaban más control de plagas y enfermedades (39\% usaba pesticidas y $54 \%$ fungicidas), que en Acatenango. En ambos paisajes el uso de prácticas AbE en la producción de granos básicos era similar, predominaba el abandono del uso del fuego como práctica de manejo, la incorporación de restos de cosecha al suelo y la labranza cero o mínima para preparar el terreno. Estas prácticas eran utilizadas tradicionalmente, excepto la labranza mínima, que en la mayoría de los casos comenzó a utilizarse en la última 
década. Los beneficios percibidos del uso de las prácticas tienen que ver principalmente con la protección frente a eventos climáticos extremos, el mejoramiento del suelo y en algunos casos el incremento de la rentabilidad de los cultivos (Figura 3). Muy pocos productores identificaban otros beneficios ecosistémicos asociados al uso de estas prácticas como el control de plagas y enfermedades, la provisión de productos o la belleza escénica.

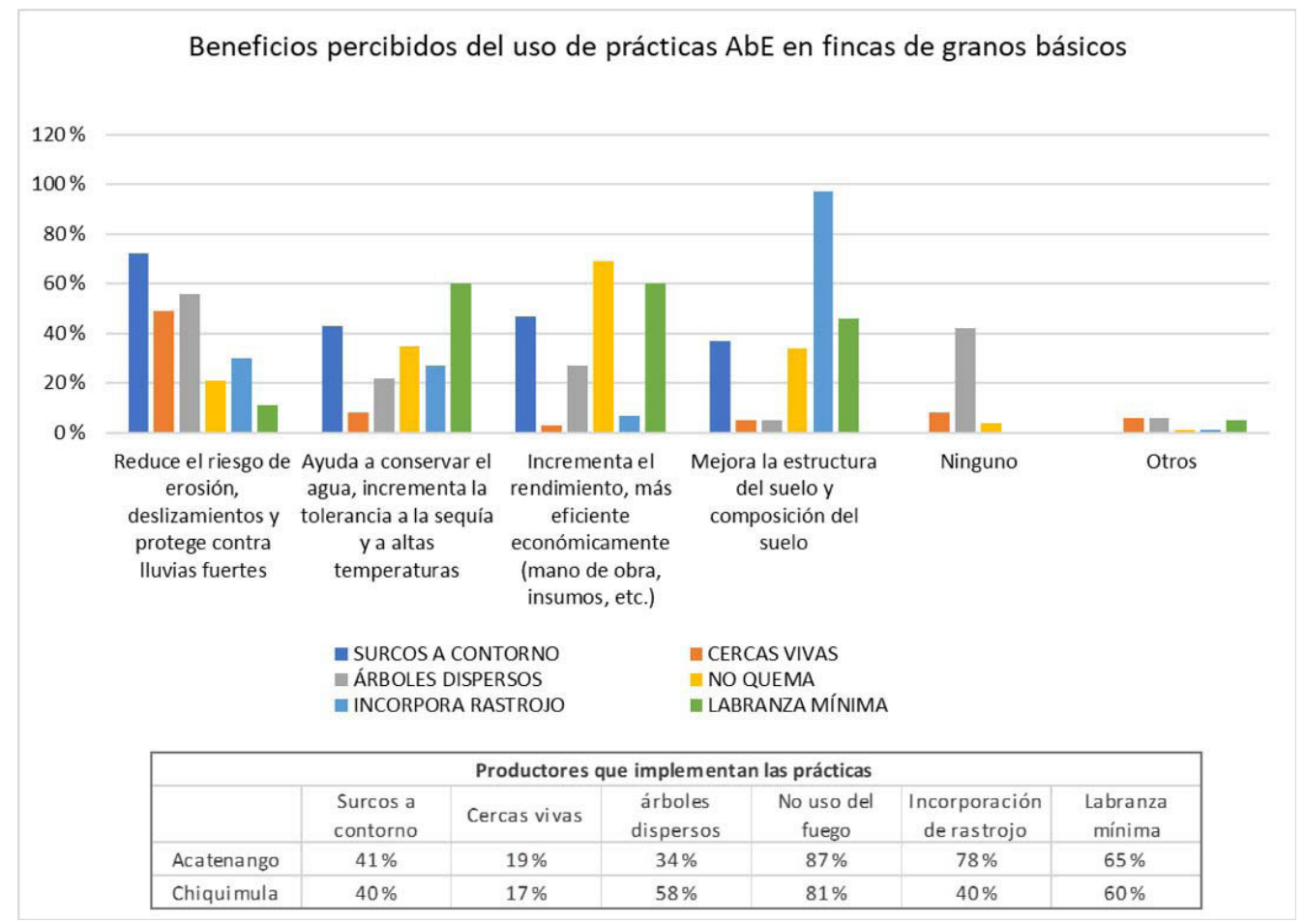

Figura 3. Beneficios del uso de las prácticas de Adaptación basada en Ecosistemas (AbE) percibidos por los productores de granos básicos en Acatenango y Chiquimula. Guatemala. 2014.

Figure 3. Benefits from using the Ecosystem-based Adaptation (EbA) practices perceived by basic grain farmers in Acatenango and Chiquimula. Guatemala. 2014.

El $89 \%$ de productores de maíz y el $73 \%$ de frijol en Acatenango identificaron problemas en sus cultivos a lo largo de la última década, mientras que en Chiquimula fueron el $95 \%$ y $93 \%$, respectivamente (Figura 4). Las plagas causaron la mayoría de los daños reportados independientemente del cultivo y el paisaje, si bien se reportó un porcentaje mayor de productores afectados en Chiquimula que en Acatenango.

\section{Percepciones de cambio climático e impactos sobre los cultivos}

En la última década (2004-2014), el $90 \%$ de los encuestados en Acatenango percibió cambios en la temperatura y el $86 \%$ en la precipitación, mientras que en Chiquimula el $88 \%$ reportaron cambios en temperatura y el $96 \%$ en precipitación. Entre los que percibieron cambios en el clima (Cuadro 4), la percepción de aumento de temperatura fue generalizada, mientras que los cambios y tendencias de precipitación percibidas variaron entre los paisajes. En Acatenango no hubo consenso sobre la variación en la cantidad de lluvia total (28\% reportó incremento y $46 \%$ 


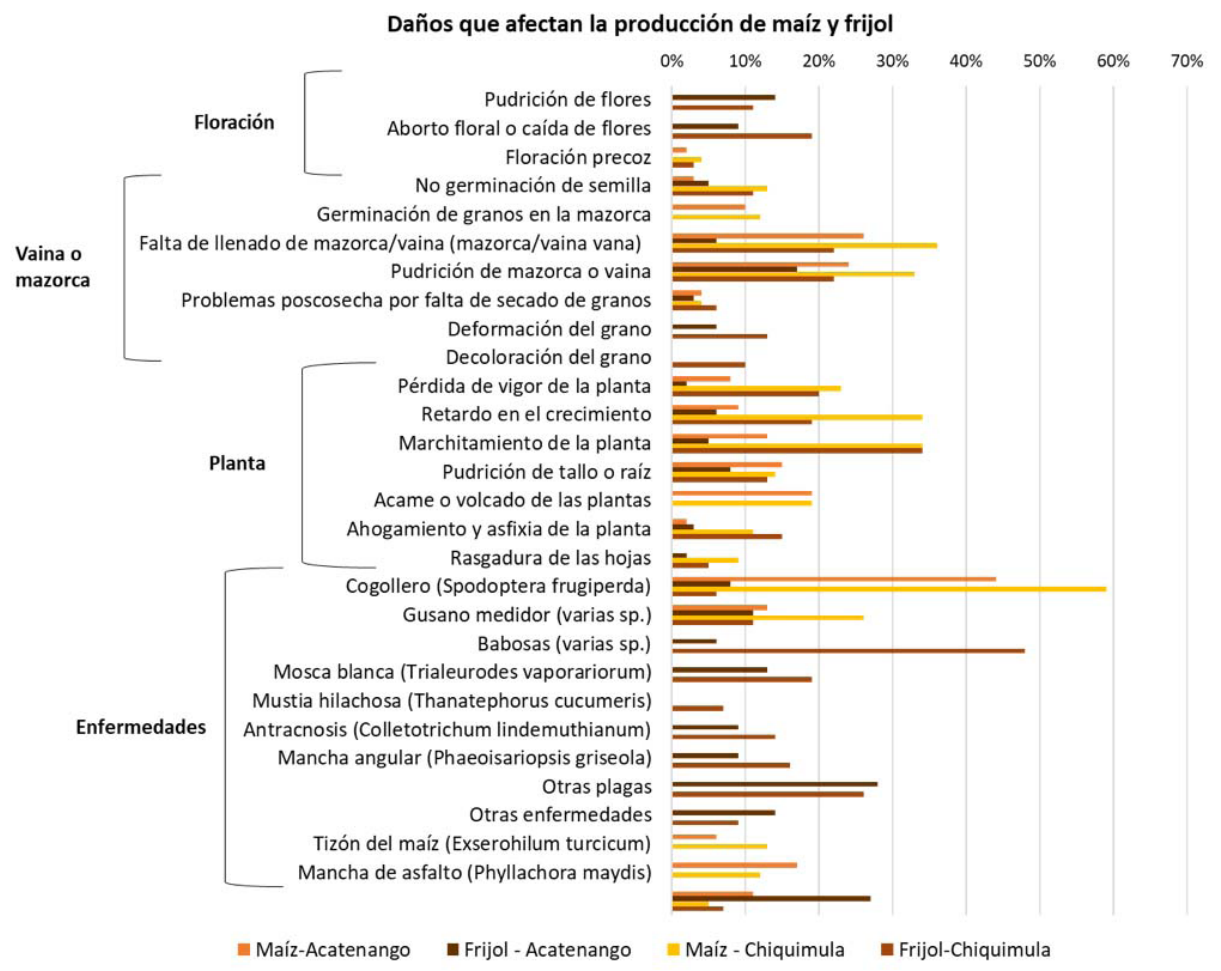

Figura 4. Problemas de producción reportados por los productores de granos básicos de Acatenango $\left(\mathrm{n}_{\text {maíz }}=89, \mathrm{n}_{\text {frijol }}=64\right)$ y Chiquimula $\left(\mathrm{n}_{\text {maiz }}=114, \mathrm{n}_{\text {frijol }}=108\right)$, Guatemala. 2004-2014.

Figure 4. Production problems reported by basic grain farmers in Acatenango $\left(\mathrm{n}_{\text {maize }}=89, \mathrm{n}_{\text {beans }}=64\right)$ and Chiquimula ( $\mathrm{n}_{\text {maize }}=114$, $\mathrm{n}_{\text {beans }}=108$ ), Guatemala. 2004-2014.

reducción), no obstante, la mayoría de la población coincidió en que hubo fluctuaciones en los patrones de lluvia que generaron incertidumbre para su producción agrícola (56 \%). En Chiquimula, la mayoría de los productores (85 \%)

Cuadro 4. Percepciones de los productores sobre cambios en el clima local observados en la última década (2004-2014), en los paisajes de Acatenango y Chiquimula, Guatemala.

Table 4. Changes perceived by farmers in local climate during the last ten years (2004-2014) in Acatenango and Chiquimula landscapes, Guatemala.

\begin{tabular}{lcc}
\hline & Acatenango & Chiquimula \\
\hline Productores que perciben cambio en temperatura & $\mathbf{N = 1 3 2}$ & $\mathbf{N = 1 0 1}$ \\
Ha incrementado & $96 \%$ & $94 \%$ \\
Ha disminuido & $2 \%$ & $0 \%$ \\
Mayor rango de variación de temperatura & $6 \%$ & $7 \%$ \\
Menor rango de variación de temperatura & $5 \%$ & $3 \%$ \\
\hline Productores que perciben cambio en precipitación & $\mathbf{N = 1 2 7}$ & $\mathbf{N = 1 1 0}$ \\
Llueve menos & $46 \%$ & $85 \%$ \\
Cambio en los patrones de lluvia & $56 \%$ & $35 \%$ \\
Llueve más intenso & $31 \%$ & $8 \%$ \\
Llueve más & $28 \%$ & $7 \%$ \\
\hline
\end{tabular}

$\mathrm{N}=$ tamaño de la muestra / $\mathrm{N}=$ sample size. 
reportaron que en la actualidad llueve menos, y algunos de ellos también identificaron cambios en los patrones de precipitación (Cuadro 4).

El $74 \%$ de los agricultores encuestados en Acatenango y el $90 \%$ en Chiquimula consideraron que los cambios del clima afectaron negativamente la producción de sus cultivos en los últimos años. En ambos paisajes consideraron que el aumento de temperatura y la variabilidad de precipitaciones fueron los factores que afectaron más la producción de café, con diferencias entre paisajes (Cuadro 5). En Chiquimula, más del $60 \%$ de los productores vinculó el aumento de temperaturas, la reducción de la precipitación y las sequías más intensas y prolongadas con impactos negativos en sus cultivos, especialmente en maíz. En Acatenango, fueron menos los que relacionaron los cambios del clima con los efectos negativos en los cultivos, y no existió un consenso sobre el tipo de cambio en las lluvias.

Cuadro 5. Cambios del clima que afectan negativamente la producción, según percepciones de los productores de Acatenango y Chiquimula, Guatemala. 2004-2014.

Table 5. Changes in climate that negatively affect crop production, based on the perceptions of smallholder farmers of Acatenango and Chiquimula, Guatemala. 2004-2014.

\begin{tabular}{|c|c|c|c|c|c|c|}
\hline \multirow{3}{*}{ Cambios del clima que afectan la producción } & \multicolumn{3}{|c|}{ Acatenango } & \multicolumn{3}{|c|}{ Chiquimula } \\
\hline & Café & Maíz & Frijoles & Café & Maíz & Frijoles \\
\hline & $\mathrm{N}=73$ & $\mathrm{~N}=\mathbf{5 8}$ & $N=44$ & $\mathbf{N}=8$ & $\mathrm{~N}=99$ & $\mathrm{~N}=85$ \\
\hline Incremento de temperatura & $78 \%$ & $53 \%$ & $39 \%$ & $50 \%$ & $73 \%$ & $61 \%$ \\
\hline Más lluvia & $23 \%$ & $28 \%$ & $36 \%$ & $0 \%$ & $1 \%$ & $5 \%$ \\
\hline Menos lluvia anual & $37 \%$ & $24 \%$ & $11 \%$ & $38 \%$ & $79 \%$ & $49 \%$ \\
\hline Lluvia concentrada en menos días (más intensa) & $30 \%$ & $19 \%$ & $25 \%$ & $0 \%$ & $6 \%$ & $20 \%$ \\
\hline Sequías más intensas & $10 \%$ & $3 \%$ & $7 \%$ & $0 \%$ & $25 \%$ & $27 \%$ \\
\hline Sequías más prolongadas & $15 \%$ & $5 \%$ & $5 \%$ & $13 \%$ & $29 \%$ & $20 \%$ \\
\hline
\end{tabular}

Se muestran resultados $>10 \%$ de respuestas / Only results with $>10 \%$ are shown. $\mathrm{N}=$ tamaño de la muestra / $\mathrm{N}=$ sample size.

\section{Cambios de manejo en respuesta a los cambios percibidos en temperatura y lluvia}

Aunque la gran mayoría de los pequeños productores percibieron cambios del clima de su región en los últimos diez años y reportaron impactos en sus cosechas a causa de estos, solamente una parte de los productores implementó medidas para adaptar sus sistemas productivos. En Acatenango, el $51 \%$ de los productores encuestados realizaron cambios en el manejo de sus fincas, tratando de adaptar sus cultivos a las nuevas condiciones climáticas, mientras que en Chiquimula solo el $24 \%$ reaccionaron a los cambios del clima. En ambos paisajes, la reacción fue mayor en quienes producían café o café y granos básicos, comparado con aquellos de solamente producción de granos básicos. Las prácticas de adaptación reportadas con más frecuencia fueron plantar árboles dentro de la finca, sembrar nuevos cultivos y cambio del calendario agrícola, que incluye cambios en las fechas de siembra, de manejo fitosanitario o de otros tratamientos culturales que se realizaba al cultivo (Cuadro 6). Algunas prácticas, como la siembra de árboles, se realizaba mayormente en respuesta a los cambios observados en temperatura, mientras que otras, como la conservación de suelos, se relacionaron a cambios observados en precipitación (Cuadro 6). 
Cuadro 6. Variación en las prácticas de manejo de la finca en respuesta a los cambios percibidos en temperatura y precipitación en Acatenango y Chiquimula, Guatemala. 2004-2014.

Table 6. Changes in farm management practices in response to perceived changes in climate in Acatenango y Chiquimula, Guatemala. 2004-2014.

\begin{tabular}{|c|c|c|c|c|c|c|c|c|c|c|}
\hline & \multicolumn{6}{|c|}{ Acatenango } & \multicolumn{4}{|c|}{ Chiquimula } \\
\hline & \multicolumn{2}{|c|}{ Café } & \multicolumn{2}{|c|}{ Granos básicos } & \multicolumn{2}{|c|}{$\begin{array}{l}\text { Café y granos } \\
\text { básicos }\end{array}$} & \multicolumn{2}{|c|}{ Granos básicos } & \multicolumn{2}{|c|}{$\begin{array}{c}\text { Café y granos } \\
\text { básicos }\end{array}$} \\
\hline & $\mathbf{T}$ & $\mathbf{P}$ & $\mathbf{T}$ & $\mathbf{P}$ & $\mathbf{T}$ & $\mathbf{P}$ & $\mathbf{T}$ & $\mathbf{P}$ & $\mathbf{T}$ & $\mathbf{P}$ \\
\hline N productores & 27 & 20 & 5 & 9 & 24 & 23 & 12 & 12 & 7 & 7 \\
\hline$\%$ del total & $48 \%$ & $36 \%$ & $14 \%$ & $24 \%$ & $43 \%$ & $41 \%$ & $12 \%$ & $12 \%$ & $41 \%$ & $41 \%$ \\
\hline \multicolumn{11}{|l|}{$\begin{array}{c}\text { Cambios en el manejo de la finca en } \\
\text { respuesta al cambio climático }\end{array}$} \\
\hline Plantar árboles dentro de la finca & $59 \%$ & $30 \%$ & $0 \%$ & $0 \%$ & $42 \%$ & $22 \%$ & $25 \%$ & $33 \%$ & $43 \%$ & $14 \%$ \\
\hline $\begin{array}{l}\text { Cambiar las prácticas de manejo del } \\
\text { cultivo (respuesta general) }\end{array}$ & $11 \%$ & $30 \%$ & $20 \%$ & $22 \%$ & $21 \%$ & $26 \%$ & $50 \%$ & $8 \%$ & $14 \%$ & $43 \%$ \\
\hline Sembrar cultivos que antes no sembraba & $19 \%$ & $10 \%$ & $40 \%$ & $0 \%$ & $0 \%$ & $4 \%$ & $8 \%$ & $0 \%$ & $14 \%$ & $29 \%$ \\
\hline Cambiar el calendario agrícola & $0 \%$ & $10 \%$ & $20 \%$ & $22 \%$ & $0 \%$ & $9 \%$ & $17 \%$ & $17 \%$ & $0 \%$ & $14 \%$ \\
\hline $\begin{array}{l}\text { Introducir prácticas de manejo y } \\
\text { conservación de suelo }\end{array}$ & $7 \%$ & $35 \%$ & $0 \%$ & $22 \%$ & $0 \%$ & $22 \%$ & $0 \%$ & $8 \%$ & $14 \%$ & $14 \%$ \\
\hline Utilizar más fertilizantes & $11 \%$ & $15 \%$ & $0 \%$ & $11 \%$ & $25 \%$ & $0 \%$ & $0 \%$ & $8 \%$ & $0 \%$ & $0 \%$ \\
\hline $\begin{array}{l}\text { Utilizar más pesticidas, herbicidas y } \\
\text { fungicidas }\end{array}$ & $7 \%$ & $0 \%$ & $0 \%$ & $22 \%$ & $8 \%$ & $0 \%$ & $0 \%$ & $0 \%$ & $0 \%$ & $0 \%$ \\
\hline $\begin{array}{l}\text { Utilizar menos pesticidas, herbicidas y } \\
\text { fungicidas }\end{array}$ & $0 \%$ & $0 \%$ & $0 \%$ & $11 \%$ & $4 \%$ & $4 \%$ & $0 \%$ & $0 \%$ & $14 \%$ & $0 \%$ \\
\hline $\begin{array}{l}\text { Cambiar la variedad de cultivos } \\
\text { sembrados }\end{array}$ & $0 \%$ & $5 \%$ & $20 \%$ & $0 \%$ & $0 \%$ & $17 \%$ & $0 \%$ & $0 \%$ & $0 \%$ & $0 \%$ \\
\hline Regar cultivos & $0 \%$ & $0 \%$ & $0 \%$ & $0 \%$ & $8 \%$ & $0 \%$ & $8 \%$ & $8 \%$ & $0 \%$ & $0 \%$ \\
\hline Dejar de cultivar ciertas zonas de la finca & $0 \%$ & $0 \%$ & $20 \%$ & $0 \%$ & $0 \%$ & $4 \%$ & $0 \%$ & $8 \%$ & $14 \%$ & $0 \%$ \\
\hline Cortar árboles dentro de la finca & $15 \%$ & $5 \%$ & $0 \%$ & $0 \%$ & $0 \%$ & $4 \%$ & $0 \%$ & $0 \%$ & $0 \%$ & $0 \%$ \\
\hline $\begin{array}{l}\text { Introducir prácticas de manejo y } \\
\text { conservación de agua }\end{array}$ & $0 \%$ & $5 \%$ & $0 \%$ & $11 \%$ & $0 \%$ & $4 \%$ & $0 \%$ & $0 \%$ & $0 \%$ & $0 \%$ \\
\hline Diversificar la producción en la finca & $0 \%$ & $5 \%$ & $0 \%$ & $0 \%$ & $0 \%$ & $4 \%$ & $0 \%$ & $0 \%$ & $0 \%$ & $0 \%$ \\
\hline Utilizar menos fertilizantes & $0 \%$ & $0 \%$ & $0 \%$ & $0 \%$ & $0 \%$ & $4 \%$ & $0 \%$ & $0 \%$ & $0 \%$ & $0 \%$ \\
\hline Restaurar áreas degradadas en la finca & $4 \%$ & $0 \%$ & $0 \%$ & $0 \%$ & $0 \%$ & $0 \%$ & $0 \%$ & $0 \%$ & $0 \%$ & $0 \%$ \\
\hline Introducir animales que antes no tenía & $0 \%$ & $0 \%$ & $0 \%$ & $0 \%$ & $0 \%$ & $0 \%$ & $0 \%$ & $0 \%$ & $0 \%$ & $0 \%$ \\
\hline Aumentar la cantidad de animales & $0 \%$ & $0 \%$ & $0 \%$ & $0 \%$ & $0 \%$ & $0 \%$ & $0 \%$ & $0 \%$ & $0 \%$ & $0 \%$ \\
\hline Reducir la cantidad de animales & $0 \%$ & $0 \%$ & $0 \%$ & $0 \%$ & $0 \%$ & $0 \%$ & $0 \%$ & $0 \%$ & $0 \%$ & $0 \%$ \\
\hline Otros & $4 \%$ & $15 \%$ & $20 \%$ & $11 \%$ & $12 \%$ & $0 \%$ & $0 \%$ & $0 \%$ & $0 \%$ & $0 \%$ \\
\hline $\begin{array}{l}\text { Número de cambios de manejo } \\
\text { implementados por finca (DE)* }\end{array}$ & \multicolumn{2}{|c|}{$1,9(1,0)$} & \multicolumn{2}{|c|}{$1,8(1,1)$} & \multicolumn{2}{|c|}{$1,5(0,8)$} & \multicolumn{2}{|c|}{$1,0(0,0)$} & \multicolumn{2}{|c|}{$1,3(0,46)$} \\
\hline
\end{tabular}

* Promedio, sin contar respuesta general / Mean, excluding general answer.

DE: desviación estándar / DE: standard deviation. 


\section{Discusión}

Los pequeños productores agrícolas encuestados en Guatemala son vulnerables al cambio climático por sus condiciones socioeconómicas y su exposición a los cambios de temperatura y precipitación. En los últimos años, los productores percibieron cambios en el clima de su región y comenzaron a experimentar los efectos de dichos cambios en sus sistemas productivos de pequeña escala, sin embargo, eran pocos los que estaban implementando acciones orientadas a mitigar el impacto de las variaciones ambientales en sus cultivos. Es necesario el apoyo gubernamental de tipo legislativo, técnico y financiero para favorecer la adaptación de los sistemas agrícolas de pequeña escala frente al cambio climático.

Tanto en Acatenango como en Chiquimula, la mayor parte de los pequeños productores percibieron cambios en el clima en la última década, principalmente incremento de la temperatura, cambios en los patrones de distribución de lluvia y reducción de lluvia total. Los cambios en el clima percibidos por los productores coinciden con algunas tendencias del clima identificadas en la región, incluyendo el incremento de temperaturas y la incertidumbre respecto a los cambios de lluvia (Magrin et al., 2014). Estudios realizados en la región mesoamericana en los últimos años, mostraron una preocupación creciente de los productores por el cambio climático frente a otros problemas que enfrentan (Tucker et al., 2010; Eakin et al., 2014; Zuluaga et al., 2015; Donatti et al., 2018) y en mayor medida por los eventos hidrometeorológicos extremos (Cruz-Bello et al., 2011; Eakin et al., 2011; Frank et al., 2011). En línea con las percepciones de los productores guatemaltecos, los caficultores nicaragüenses reportaron incrementos de temperatura, cambios en la frecuencia o estacionalidad de las lluvias, sequías e inundaciones más intensas que en la década anterior (Baca et al., 2014; Zuluaga et al., 2015), así como eventos extremos más frecuentes (Baca et al., 2014).

El incremento de la temperatura, en combinación con los cambios en la cantidad y distribución de las lluvias y la incidencia de eventos hidrometeorológicos extremos, dificultan la planificación de las actividades agrícolas y afectan a los productores de múltiples formas (daño a infraestructura agrícola y vial, proliferación de plagas y enfermedades, etc.). Las variaciones en el clima afectan negativamente el desarrollo y la productividad de los cultivos de café y granos básicos de forma directa (Gay et al., 2006; Gourdji et al., 2015; Chaves-Barrantes y Gutiérrez-Soto, 2017), así como a la proliferación de plagas y enfermedades a consecuencia de estas (Altamirano, 2012; Avelino et al., 2015). Más del $80 \%$ de los encuestados relacionaron los cambios en el clima con pérdidas de producción, aunque la incidencia negativa de los cambios varió entre paisajes y cultivos. En línea con este resultado, caficultores de México reportaron pérdidas directas e indirectas en su producción, asociadas a los cambios del clima, en particular a consecuencia del incremento en la frecuencia e intensidad de los eventos extremos, lluvias fuertes, exceso de humedad y temperaturas extremas (Frank et al., 2011).

A pesar de que gran mayoría de los pequeños productores percibieron cambios del clima de su región en los últimos diez años, y relacionaron dichos cambios con impactos en sus cosechas de café, maíz y frijol, solo una parte de ellos había implementando medidas para adaptar sus sistemas productivos. Los que cultivaban café (solo o con granos básicos) reaccionaron más a las variaciones percibidas, que los productores que solamente cultivaban granos básicos. Esto podría deberse a que los caficultores tenían mayor acceso a recursos financieros y asistencia técnica que los productores de granos básicos (Holland et al., 2017) y, por lo tanto, pueden implementar medidas de adaptación con más facilidad. En Acatenango, la adaptación en café se vio más motivada por el incremento de temperatura, y en el caso de los granos básicos por la modificación de los patrones de lluvia. Esto puede deberse a que los productores eran más conscientes de ciertas alteraciones en función de cómo estasafectaban a sus cultivos principales y los cambios asociados a determinadas etapas fenológicas de sus cultivos. En este aspecto, el calendario agrícola de los granos básicos ha estado sincronizado con los periodos de lluvia, lo que explica la mayor preocupación de los productores de maíz y frijol en este aspecto, mientras que las altas temperaturas afectan la floración y por lo tanto, la producción del café (Altamirano, 2012), sin embargo, ambos cultivos se ven también afectados por otras variables climáticas. 
Entre las prácticas implementadas con más frecuencia estaban la incorporación de árboles en los cultivos, la diversificación de cultivos, el cambio del calendario agrícola y prácticas de conservación de suelos. A excepción del cambio de calendario agrícola, lo que los productores más estaban implementando eran prácticas de Adaptación basada en Ecosistemas (AbE), aunque las prácticas utilizadas variaron entre paisajes y cultivos. Además, ciertas prácticas se utilizaban en mayor medida en respuesta al incremento de temperatura, como la siembra de árboles, por su contribución a regular la temperatura, y otras en respuesta a la variación de las lluvias, como la conservación de suelos, por el papel que cumple tanto en mantenimiento de la humedad en épocas secas como de mantenimiento del suelo en episodios de lluvias intensas. Algunas prácticas eran utilizadas principalmente por productores de granos básicos, como el cambio del calendario agrícola, el cambio de variedades y el descanso de la tierra, que tienen que ver con prácticas tecnológicas. El incremento del uso de agroquímicos como estrategia de adaptación era más frecuente en Acatenango, y en algunos casos respondía a cambios en temperatura y en otros a cambios en lluvias. Al igual que en otros países centroamericanos, los productores de café tendieron a realizar más cambios de manejo en sus fincas que los de granos básicos (Viguera et al., 2018). Sin embargo, todos los grupos de productores implementaban pocos cambios, entre 1 y 2 en promedio. Esta cifra es baja considerando que las prácticas generalmente se pueden implementar de forma simultánea y pueden tener efectos adaptativos diferentes (por ejemplo, protección frente a extremos de temperatura, vientos o lluvias fuertes).

A pesar del escaso número de cambios de manejo implementados por los encuestados, los resultados indicaron las prácticas preferidas en cada contexto, así como aquellas que requieren más apoyo para su implementación. En este último grupo se encuentran prácticas de reconocida utilidad para pequeños productores que no estaban siendo implementadas, tales como la diversificación de la producción o la transición a sistemas menos dependientes de agroquímicos, así como mejoras tecnológicas que usualmente escapan del alcance de pequeños productores guatemaltecos por la falta de recursos económicos y acceso a asistencia técnica (MAGA, 2015). Esta información, junto con la identificación de los productores más vulnerables, los pronósticos de cambio para la región y el impacto esperado sobre los servicios ecosistémicos permitirán a los tomadores de decisión planificar los procesos de adaptación con base en las necesidades específicas del sector (Donatti et al., 2017). En sistemas agrícolas de pequeña escala, la adaptación debe fomentarse por medio de asistencia técnica específica, enfocada en las necesidades de adaptación de cada región y sistema productivo. Además, se necesita un marco legal que promueva el uso de prácticas de adaptación en sistemas productivos de gran y pequeña escala, e incentivos especialmente orientados a los pequeños productores que no cuentan con los medios necesarios para implementar acciones de adaptación necesarias. El apoyo de mecanismos financieros es clave para pequeños productores, y podría materializarse por medio de esquemas de pago por servicios ambientales por actividades que promuevan la conservación y la conectividad a nivel de paisaje, créditos de bajo interés y facilidades para acceder a mercados diferenciados, donde los requerimientos de certificación limitan el acceso de los pequeños productores.

La adaptación del sector agrícola es clave para el cumplimiento de los compromisos que los países centroamericanos han adquirido con la comunidad internacional (Acuerdo de París, entre otros) y para el cumplimiento de los objetivos de desarrollo sostenible (en particular para la reducción de la pobreza y la inseguridad alimentaria). En este aspecto, la Adaptación basada en Ecosistemas constituye una oportunidad para facilitar la adaptación de los pequeños productores y contribuir a las metas de adaptación y mitigación (Pramova et al., 2012). Por medio de la $\mathrm{AbE}$, los pequeños productores pueden incrementar su resiliencia socioeconómica y ambiental de sus actividades productivas, a la vez que contribuyen a mitigar gases de efecto invernadero, la conectividad y la provisión de servicios ecosistémicos a escala de paisaje. Este estudio indica además, que los productores guatemaltecos tenían acceso y cierto conocimiento de estas prácticas, dado que algunos de ellos ya las implementaban en sus fincas y percibían los beneficios de su uso. Tanto AbE como otras medidas de adaptación pueden fomentarse por medio de los acuerdos y compromisos internacionales, y también con el apoyo del sector privado. De cualquier forma, la adaptación del sector agrícola en Guatemala debe ser una prioridad a nivel nacional por la inminencia del cambio climático y la severidad de los impactos esperados. 


\section{Conclusiones}

Los cambios en las condiciones climáticas en la región centroamericana pueden reducir la productividad y rentabilidad de los cultivos, e incrementar el impacto de plagas y enfermedades, entre otros efectos directos e indirectos a corto plazo. En los paisajes estudiados, los productores habían percibido cambios en el clima que asociaron a impactos negativos sobre su producción de café y/o granos básicos, sin embargo, los esfuerzos de adaptación eran escasos, ya que tanto caficultores como productores de granos básicos solo implementaban entre una y dos medidas de adaptación en respuesta a los cambios observados. Los pequeños caficultores guatemaltecos presentaban mayores tasas de respuesta al cambio climático que los pequeños productores de granos básicos, diferencia que podrían deberse al mayor acceso a asistencia técnica y recursos económicos con los que contaban los caficultores. Las prácticas implementadas variaban entre paisajes y tipos de cultivo, siendo la práctica de adaptación más frecuente la siembra de árboles, bien sea como sombra del café, o de forma dispersa en los cultivos de maíz y frijol, y las prácticas menos comunes eran aquellas que tienen que ver con la diversificación de actividades productivas.

Ante los pronósticos de cambio climático, y con las limitantes que afrontan los pequeños productores, es crítico que los gobiernos de la región centroamericana, en colaboración con donantes, técnicos y el sector privado, apoyen a los pequeños productores agrícolas a llevar a cabo acciones de adaptación al cambio climático. Para esto, los pequeños agricultores requieren asistencia técnica especializada, así como apoyo financiero y legislativo para implementar las medidas de adaptación apropiadas para sus sistemas productivos y su contexto local. A nivel nacional, se puede promover el uso de prácticas de adaptación al cambio climático apropiadas por medio de la elaboración de políticas que atiendan las necesidades de los pequeños productores agrícolas, la promoción de planes y estrategias nacionales preventivas frente a riesgos esperados, y el fortalecimiento de las capacidades de las familias para responder a los cambios y los impactos esperados.

\section{Agradecimientos}

Los autores agradecen el apoyo de Tabaré Capitán en el diseño e implementación de la encuesta a hogares, de Marie Cadudal en la reestructuración y revisión de los cuadros y figuras del documento, de Ahías Steller por el apoyo en la integración de comentarios y sugerencias y del equipo que participó en la recolección de datos y a la preparación de los mismos.

\section{Literatura citada}

Altamirano, J.A. 2012. Influencia de la variabilidad climática sobre la producción de café (Coffea arabica L.) en Honduras. Tesis MSc., CATIE, Turrialba, CRI.

Alpizar, F., C.A. Harvey, M. Saborío-Rodríguez, B. Viguera, M.R. Martínez-Rodríguez, and R. Vignola. 2019. Household survey of climate change perception and adaptation strategies of smallholder coffee and basic grain farmers in Central America 20042014. UK Data Service, Colchester, Essex, GBR. http://reshare.ukdataservice.ac.uk/853252/ (consultado 30 mar. 2019).

Avelino, J., M. Cristancho, S. Georgiou, P. Imbach, L. Aguilar, G. Bornemann, P. Läderach, F. Anzueto, A.J. Hruska, and C. Morales. 2015. The coffee rust crises in Colombia and Central America (2008-2013): impacts, plausible causes and proposed solutions. Food Secur. 7:303-321. doi:10.1007/s12571-015-0446-9

Baca, M., P. Läderach, J. Haggar, G. Schroth, and O. Ovalle. 2014. An integrated framework for assessing vulnerability to climate change and developing adaptation strategies for coffee growing families in Mesoamerica. PLoS ONE 9(2):e88463. doi:10.1371/journal.pone.0088463 
Buppert, T., and A. McKeehan. 2013. Guidelines for applying free, prior and informed consent: a manual for conservation international. Conservation International, VA, USA.

Burke, M., and D. Lobell, 2010. Food security and adaptation to climate change: What do we know? In: D. Lobell, and M. Burke, editors, Climate Change and Food Security. Advances in Global Change Research. Vol. 37. Springer, Dordrecht, HOL. p. 133-153. doi:10.1007/978-90-481-2953-9_8

CBD (Secretariat of the Convention on Biological Diversity). 2009. Connecting biodiversity and climate change mitigation and adaptation: Key messages from the report of the second Ad Hoc technical expert group on biodiversity and climate change. CBD, Montreal, CAN. https://www.cbd.int/doc/publications/ahteg-brochure-en.pdf (accessed Aug. 9, 2017).

CEPAL (Comisión Económica para América Latina y el Caribe), y CAC/SICA (Consejo Agropecuario Centroamericano/ Sistema de la Integración Centroamericano). 2014. Impactos potenciales del cambio climático sobre el café en Centroamérica. CEPAL, MEX.

Chaves-Barrantes, N.F., y M.V. Gutiérrez-Soto. 2017. Respuestas al estrés por calor en los cultivos. II. Tolerancia y tratamiento agronómico. Agron. Mesoam. 28:255-271. doi:10.15517/am.v28i1.21904

Climate-Data.org. 2018. Climate: Guatemala. Climate-Data.org. https://en.climate-data.org/country/229/ (accessed Mar. 14, 2018).

Conway, G. 2011. On being a smallholder. Presented at: Conference on New Directions for Smallholder Agriculture. IFAD, Rome, ITA, 24-25 Jan.

Cruz-Bello, G.M., H. Eakin, H. Morales, and J.F. Barrera. 2011. Linking multi-temporal analysis and community consultation to evaluate the response to the impact of hurricane stan in coffee areas of Chiapas, Mexico. Nat. Hazards 58:103-116. doi:10.1007/s11069-010-9652-0

Donatti, C.I., C.A. Harvey, M.R. Martinez-Rodriguez, R. Vignola, and C.M. Rodríiguez. 2018. Vulnerability of smallholder farmers to climate change in Central America and Mexico: current knowledge and research gaps. Climate Dev. $2018: 5529$. doi:10.1080/17565529.2018.1442796

Donatti, C.I., C.A. Harvey, M.R. Martinez-Rodriguez, R. Vignola, and C.M. Rodríguez. 2017. What information do policy makers need to develop adaptation plans for smallholder farmers? The case of Central America and Mexico. Climatic Change 141:107-121. doi:10.1007/s10584-016-1787-x

Eakin, H., L.A. Bojórquez-Tapia, R. Monterde, E. Castellanos, and J.Haggar. 2011 . Adaptive capacity and social-environmental change: Theoretical and operational modeling of smallholder coffee systems response in Mesoamerican pacific rim. Environ. Manag. 47:352-367. doi:10.1007/s00267-010-9603-2

Eakin, H., C.M. Tucker, E. Castellanos, R. Diaz-Porras, J.F. Barrera, and H. Morales. 2014. Adaptation in a multi-stressor environment: perceptions and responses to climatic and economic risks by coffee growers in Mesoamerica. Environ. Dev. Sustain. 16:123-139. doi:10.1007/s10668-013-9466-9

Eitzinger, A., P. Läderach, K. Sonder, A. Schmidt, G. Sain, S. Beebe, B. Rodríguez, M. Fisher, P. Hicks, C. Navarrete-Frías, and A. Nowak. 2013. Tortillas on the roaster: Central America's maize-bean systems and the changing climate. Policy Brief. CATIE, and CI, Turrialba, CRC.

Frank, E., H. Eakin, and D. López-Carr. 2011. Social identity, perception and motivation in adaptation to climate risk in the coffee sector of Chiapas, Mexico. Global Environ. Change 21:66-76. doi:10.1016/j.gloenvcha.2010.11.001

Galindo, L.M., J. Samaniego, J.E. Alatorre, y J.F. Carbonell. 2014. Procesos de adaptación al cambio climático. Análisis de América Latina. Documentos de Proyecto (LC/W.647). CEPAL, Santiago, CHI. https://repositorio.cepal.org/bitstream/ handle/11362/37613/1/S1500005_es.pdf (consultado 27 abr. 2018). 
Gay, C., F. Estrada, C. Conde, H. Eakin, and L. Villers. 2006. Potential impacts of climate change on agriculture: A case of study of coffee production in Veracruz, Mexico. Climatic Change 79:259-288. doi:10.1007/s10584-006-9066-X

Gourdji, S., P. Läderach, A. Martinez, C. Zelaya, and D.B. Lobell. 2015. Historical climate trends, deforestation, and maize and bean yields in Nicaragua. Agric. For. Meteorol. 200:270- 28. doi:10.1016/j.agrformet.2014.10.002

Hannah, L., C.I. Donatti, C.A. Harvey, E. Alfaro, D.A. Rodriguez, C. Bouroncle, E. Castellanos, F. Diaz, E. Fung, H.G. Hidalgo, P. Imbach, P. Läderach, J.P. Landrum, and A.L. Solano. 2017. Regional modeling of climate change impacts on smallholder agriculture and ecosystems in Central America. Climatic Change 141:29-45. doi:10.1007/s10584-016-1867.

Holdridge, L.R. 1947. Determination of world plant formations from simple climatic data. Science 105:367-368. doi:10.1126/ science.105.2727.367

Holland, M.B., S.Z. Shamer, P. Imbach, J.C. Zamora, C. Medellin Moreno, E.J.L. Hidalgo, C.I. Donatti, M.R. MartínezRodríguez, and C.A. Harvey. 2017. Mapping adaptive capacity and smallholder agriculture: applying expert knowledge at the landscape scale. Climatic Change 141:139-153. doi:10.1007/s10584-016-1810-2

Imbach, P., M. Beardsley, C. Bouroncle, C. Medellin, P. Läderach, H. Hidalgo, E. Alfaro, J. Van-Etten, R. Allan, D. Hemming, R. Stone, L. Hannah, and C.I. Donatti. 2017. Climate change, ecosystems and smallholder agriculture in Central America: an introduction to the special issue. Climatic Change 141:1-12. doi:10.1007/s10584-017-1920-5

Imbach P., B. Locatelli, J.C. Zamora, E. Fung, L. Calderer, L. Molina, and P. Ciais. 2015. Climate, water and land-use changes in Central American tropical forests. In: A. Chiabai, editor, Climate change impacts on tropical forests in Central America. Routledge, NY, USA. p. 63-178.

Imbach P., L. Molina, B. Locatelli, O. Roupsard, G. Mahe, R. Neilson, L. Corrales. M., Scholze, and P. Ciais. 2012. Modeling potential equilibrium states of vegetation and terrestrial water cycle of Mesoamerica under climate change scenarios. J. Hydrometeorol. 13:665.680. doi:10.1175/JHM-D-11-023.1

IPCC. 2012. Managing the risks of extreme events and disasters to advance climate change adaptation. Cambridge University Press, Cambridge, GBR, and NY, USA.

MAGA (Dirección de Planeamiento del Ministerio de Agricultura, Ganadería y Alimentación). 2015. El agro en cifras 2014. MAGA, GUA. http://web.maga.gob.gt/diplan/download/informacion_del_sector/agro_en_cifras/El_agro_en_cifras_2014. pdf (consultado 4 may. 2018).

Magrin, G.O., J.A. Marengo, J.P. Boulanger, M.S. Buckeridge, E. Castellanos, G. Poveda, F.R. Scarano, and S. Vicuña. 2014. Central and South America. In: V.R Barros et al., editors, Climate change 2014: Impacts, adaptation, and vulnerability. Part B: Regional aspects. Contribution of working group II to the fifth assessment report of the Intergovernmental Panel on Climate Change. Cambrige University Press, GBR and NY, USA. p. 1499-1556.

MARN (Ministerio de Medio Ambiente y Recursos Naturales). 2015. Contribución prevista y determinada a nivel nacional de El Salvador Bonn, Alemania, UNFCCC. UNDP. https://info.undp.org/docs/pdc/Documents/GTM/00038379\%20Informe\%20 final\%20Proyecto.pdf (consultado 16 ago. 2018).

Morton, J.F. 2007. The impact of climate change on smallholder and subsistence agriculture. PNAS 104:19680-19685. doi:10.1073/pnas.0701855104.

Noble, I.R., S. Huq, Y.A. Anokhin, J. Carmin, D. Goudou, F.P. Lansigan, B. Osman-Elasha, and A. Villamizar. 2014. Adaptation needs and options. In: C.B. Field et al., editors, Climate change 2014: Impacts, adaptation, and vulnerability. Part A: global and sectoral aspects. Contribution of working group II to the Fifth assessment report of the Intergovernmental Panel on Climate Change. Cambridge University Press, GBR and NY, USA. p. 833-868. 
Pramova, E., B. Locatelli, M. Brockhaus, and S. Fohlmeister. 2012. Ecosystem services in the national adaptation programmes of action. Climate Policy 12:393-409. doi:10.1080/14693062.2011.647848

Tucker, C.M., H. Eakin, and E.J. Castellanos. 2010. Perceptions of risk and adaptation: coffee producers, market shocks, and extreme weather in Central America and Mexico. Global Environ. Change 20:23-32. doi:10.1016/j.gloenvcha.2009.07.006

Vignola, R., C.A Harvey, P. Bautista-Solis, J. Avelino, B. Rapidel, C. Donatti, and R. Martinez. 2015. Ecosystem-based adaptation for smallholder farmers: Definitions, opportunities and constraints. Agric. Ecosyst. Environ. 211:126-132. doi:10.1016/j. agee.2015.05.013

Viguera, B., M. R. Martínez-Rodríguez, F. Alpízar, y C.A. Harvey. 2018. Adaptación basada en ecosistemas como una opción de adaptación de la agricultura al cambio climático en Centroamérica. Policy Brief. CATIE, y CI, Turrialba, CRC.

Zuluaga, V., R.A. Labarta, and P. Läderach. 2015. Climate change adaptation: The case of coffee sector in Nicaragua. Present at: 2015 Agricultural \& Applied Economics Association and Western Agricultural Economics Association Annual Meeting, San Francisco, CA, USA. July 26-28. https://cgspace.cgiar.org/rest/bitstreams/55624/retrieve (accessed Jun. 18, 2017). 